\title{
Comparação pressórica e visual entre a facoesclerectomia profunda não penetrante e a facotrabeculectomia
}

\author{
Intraocular pressure and visual results from combined surgery: \\ phacoemulsification with non penetrating deep sclerectomy \\ and phacoemulsification with trabeculectomy
}

Juliana Almodin' ${ }^{1}$ Eduardo Cavalheiro Pedroso ${ }^{2}$, Tadeu Cvintal ${ }^{3}$, Flavia Almodin ${ }^{4}$

\section{Resumo}

Objetivo: O presente estudo tem como objetivo comparar os resultados do controle pressórico e acuidade visual (AV) entre a facoesclerectomia profunda não penetrante (FACOEPNP) e facotrabeculectomia (FACOTREC). Métodos: Estudo observacional, retrospectivo através da revisão de prontuários. Foram selecionados 148 olhos de 127 pacientes submetidos à cirurgia no período de janeiro de 1998 a janeiro de 2007 do Instituto de Oftalmologia Tadeu Cvintal em portadores de glaucoma primário de ângulo aberto (GPAA) com diagnóstico de catarata coexistente. Destes pacientes 77 eram do sexo feminino, sendo que $49(59 \%)$ foram submetidos à FACOEPNP e $28(63,6 \%)$ foram submetidos à FACOTREC. Cinquenta pacientes eram do sexo masculino sendo, 34 (41\%) submetidos à FACOEPNP e $16(36,4 \%)$ a FACOTREC. O estudo teve duração de 12 meses e os pacientes tiveram pressão intraocular (PIO) (tonômetro de Goldmann) e AV (LogMar) aferidos no pré-operatório e um ano após o procedimento cirúrgico, além do número de medicações hipotensoras oculares que foram adicionados no pósoperatório.Resultados: Os grupos se mostraram homogêneos quanto a AV pré $(\mathrm{p}=0,067)$, AV pós $(p=0,924)$ e variação da $A V(p=0,099)$. A média de variação de $A V$ no grupo FACOTREC foi de $-0,31(\mathrm{dp}=0,59)(\mathrm{p}<0,001)$, enquanto que a média de variação da AV no grupo da FACOEPNP foi de $-0,45$ (dp 0,51$)$ com $p<0,001$. A média de variação de PIO no grupo FACOEPNP de $-6,2(\mathrm{dp}=5,1)$ foi estatisticamente significante $(\mathrm{p}<0,001)$ e a média de variação de PIO no grupo FACOTREC foi de -6,6 (dp = 5,3) $(\mathrm{p}<0,001)$. Não foi encontrada diferença estatisticamente significante entre os grupos quanto ao uso de medicações na avaliação pós $(\mathrm{p}=0,398)$. Conclusão: A esclerectomia não penetrante (EPNP) combinada com cirurgia de catarata resulta em uma redução da PIO similar a encontrada na FACOTREC com o mesmo resultado com relação a melhor AV.

Descritores: Glaucoma de ângulo aberto /cirurgia; Trabeculectomia; Esclera /cirurgia; Facoemulsificação; Pressão intraocular; Acuidade visual; Terapia combinada

\footnotetext{
${ }^{1}$ Assistente do Departamento de Glaucoma do Instituto de Oftalmologia Tadeu Cvintal - São Paulo (SP), Brasil; ${ }^{2}$ Residente do Terceiro ano de oftalmologia do Instituto de Oftalmologia Tadeu Cvintal - São Paulo (SP), Brasil; ${ }^{3}$ Diretor e Chefe do Departamento de Córnea do Instituto de Oftalmologia Tadeu Cvintal - São Paulo (SP), Brasil; ${ }^{4}$ Estagiária do Instituto de Oftalmologia Tadeu Cvintal - São Paulo (SP), Brasil;

Departamento de Glaucoma do Instituto de Oftalmologia Tadeu Cvintal - São Paulo (SP), Brasil;

Recebido para publicação em: 18/3/2010 - Aceito para publicação em 13/4/2010
} 


\section{INTRODUÇÃO}

G laucoma e catarata são duas entidades que frequentemente ocorrem ao mesmo tempo, e a realização de uma cirurgia combinada é uma alternativa terapêutica que dispomos no momento. ${ }^{(1)} \mathrm{A}$ facotrec tem sido a intervenção mais popular quando se deve combinar a cirurgia de catarata e glaucoma em um mesmo ato cirúrgico, constituindo um procedimento gratificante para o paciente, combinando a reabilitação visual com menor dependência do tratamento médico antiglaucomatoso. $^{(2)}$

A EPNP tem surgido como uma alternativa a trabeculectomia convencional, com menor número de complicações pós-operatórias associadas. São cada vez mais numerosos os estudos que confirmam sua eficácia e sua segurança. ${ }^{(3)}$

A decisão de se optar por uma cirurgia combinada para a catarata e o glaucoma depende de alguns fatores individuais de cada paciente, como: PIO alvo, estágio do glaucoma e expectativa de vida. ${ }^{(4)}$

Entretanto, a EPNP não é superior a trabeculectomia (TREC) em relação ao controle da PIO, porém reduz as complicações tanto intraoperatórias como pós-operatórias. ${ }^{(5)} \mathrm{O}$ uso de implantes esclerais parece melhorar a filtração e diminuir a fibrose, porém pode aumentar o custo da cirurgia, o que em algumas ocasiões pode restringir o seu uso. ${ }^{(6)}$

O uso de antimetabólitos, que modulam a resposta cicatricial, podem ser utilizados para aumentar a taxa de sucesso, mas não é um processo livre de complicações. ${ }^{(7)}$ Há estudos que mostram o benefício da aplicação da mitomicina $\mathrm{C}$ (MMC) como um coadjuvante na cirurgia combinada de catarata e glaucoma, particularmente em pacientes com risco para a falência da cirurgia filtrante. ${ }^{(5)}$

Alguns fatores de risco conhecidos para a falência na cirurgia filtrante são: presença de inflamação, olhos submetidos à cirurgia ocular prévia, uso prolongado de medicação tópica antiglaucomatosa, raça negra e jovens. ${ }^{(7)}$

Não há ainda evidência científica suficiente para determinar qual a técnica de cirurgia combinada mais efetiva. ${ }^{(8)}$ Por esse motivo, nos propusemos a avaliar a eficácia de ambos os procedimentos.

\section{MÉTODOS}

Este foi um estudo observacional, retrospectivo através da revisão de prontuários. Foram selecionados 148 olhos de 127 pacientes submetidos à cirurgia no pe- ríodo de janeiro de 1998 a janeiro de 2007 do Instituto de Oftalmologia Tadeu Cvintal em portadores de GPAA com diagnóstico de catarata coexistente. Destes pacientes 77 eram do sexo feminino, sendo que 49 (59\%) foram submetidos à FACOEPNP e $28(63,6 \%)$ foram submetidos à FACOTREC. Cinquenta pacientes eram do sexo masculino, sendo que 34 (41\%) foram submetidos à FACOEPNP e $16(36,4 \%)$ à FACOTREC. Todos os pacientes tinham PIO não controlada com medicação antiglaucomatosa máxima tolerada ou PIO controlada clinicamente no período pré-operatório e a opção pela cirurgia combinada foi tomada a critério médico se o paciente estivesse com glaucoma em estágio avançado ou glaucoma controlado com duas ou mais medicações hipotensoras oculares; e que também necessitavam de cirurgia para a catarata. Consideramos para este estudo como catarata sendo qualquer opacidade do cristalino e GPAA como tendo alteração típica de nervo óptico (NO), e/ou alteração típica campimétrica e/ou insuficiente controle clínico da PIO. Foram excluídos do trabalho pacientes portadores de glaucoma congênito, glaucomas secundários, glaucoma primário de ângulo fechado, cirurgia ocular prévia, história de trauma ocular e descolamento de retina. O estudo teve duração de 12 meses e os pacientes tiveram PIO (tonômetro de Goldmann) e AV (LogMar) aferidos no pré-operatório e um ano após procedimento cirúrgico, além do número de medicações hipotensoras oculares que foram adicionados no pós-operatório. A cirurgia foi realizada por um cirurgião experiente do próprio serviço e a técnica cirúrgica adotada foi a seguinte:

Facotra $^{(11)}$ eculectomia:

1.Retopexia do músculo reto superior com seda 4-0;

2.Peritomia de base fórnice e hemostasia com cautério bipolar;

3.Aplicação de MMC 0,025 mg/ml por 3 minutos;

4.Irrigacão com solução salina balanceada;

5. Confecção do retalho escleral quadrangular de $4 \times 4$ $\mathrm{mm}$;

6. Incisão em córnea clara com 3,2 mm de largura;

7.Capsulorrexis e hidrodissecção do núcleo;

8.Facoemulsificação e aspiração dos restos corticais;

9. Implante de lente intraocular (LIO);

10.Retirada de um bloco de tecido escleral de aproximadante $1,5 \mathrm{~mm} \times 1,5 \mathrm{~mm}$;

11. Iridectomia com tesoura de Vannas;

12.Sutura do retalho escleral com mononylon 10-0;

13.Sutura conjuntival com mononylon 10-0;

14.Injeção subconjuntival de dexametasona e gentamicina 
Facoesclerectomia:

1. Retopexia do músculo reto superior com seda 4-0;

2. Abertura da conjuntiva e cápsula de Tenon com base fórnice;

3. Confecção do retalho escleral superficial quadrangular de $5 \times 5 \mathrm{~mm}$ com $1 / 3$ de profundidade;

4. Confecção de retalho escleral profundo triangular de base de 4 a $5 \mathrm{~mm}$ até o plano pré-ciliar;

5. Excisão do retalho profundo, retirando junto a parede externa do CS;

6. Excisão da membrana trabecular externa, que inclui a parede interna do CS e o trabeculado justacanalicular; 7. Sutura do retalho superficial com dois pontos com mononylon 10-0;

8. Sutura conjuntival com pontos separados de mononylon 10-0;

9. Incisão em córnea clara com 3,2 mm de largura;

10. Capsulorrexis e hidrodissecção do núcleo;

11. Facoemulsificação e aspiração dos restos corticais;

12. Implante de lente intraocular (LIO);

13.Injeção subconjuntival de dexametasona e gentamicina

No pós-operatório de ambas as técnicas, os pacientes foram instruídos a utilizar uma combinacão fixa de dexametasona, polimixina B e neomicina a cada 4 horas e tropicamida $1 \%$ a cada 12 horas por 4 a 6 semanas.

Foram adotados pelos autores como critérios de sucesso: sucesso absoluto: pacientes com PIO menor/ igual a $21 \mathrm{mmHg}$ sem a necessidade de tratamento clínico após o procedimento e sucesso relativo: PIO menor/igual a $21 \mathrm{mmHg}$ associado ao tratamento clínico. Os dados relativos à $\mathrm{AV}$, $\mathrm{PIO}$, número de medicações hipotensoras foram analisados no pré-operatório e 12 meses após o procedimento cirúrgico.

\section{Resultados}

Os grupos se mostraram homogêneos quanto à média de idade $(\mathrm{p}=0,153)$ e distribuição por sexo dos pacientes $(\mathrm{p}=0,614)$.

Nas avaliações de AV LogMAR foram encontrados os seguintes resultados: os grupos se mostraram homogêneos quanto à $A V$ pré $(p=0,067), A V$ pós $(p=0,924)$ e variação da $\mathrm{AV}(\mathrm{p}=0,099)$; a média de variação de $\mathrm{AV}$ no grupo FACOEPNP de -0,45 ( dp = 0,51) foi estatisticamente significante $(\mathrm{p}<0,001)$, da mesma forma que a média de variação de AV no grupo FCOTREC foi de $0,31(\mathrm{dp}=0,59)(\mathrm{p}<0,001)$.

Nas avaliações de PIO os grupos se mostraram homogêneos quanto a PIO pré $(\mathrm{p}=0,190)$, PIO pós ( $\mathrm{p}=$
0,376) e variação da PIO ( $\mathrm{p}=0,856)$; (Tabela 1$)$

A proporção de pacientes com $\mathrm{PIO} \leq 21 \mathrm{mmHg}$ foi semelhante nos grupos nas avaliações pré $(p=0,144)$ e pós $(p=0,264)$;

A média de variação de PIO no grupo FACOEPNP de $-6,2(\mathrm{dp}=5,1)$ foi estatisticamente significante $(\mathrm{p}<$ $0,001)$, assim como a média de variação de PIO no grupo FACOTREC de -6,6 (dp = 5,3) $(\mathrm{p}<0,001)$.

Não foi encontrada diferença estatisticamente significante entre os grupos quanto ao uso de medicações na avaliação pós $(\mathrm{p}=0,398)$ e não foi encontrada diferença estatisticamente significante entre os grupos quanto à distribuição dos resultados de eficácia dos procedimentos $(\mathrm{p}=0,253)$. (Tabela 2$)$

\section{Dıscussão}

A FACOTREC tem sido o procedimento eleito para cirurgia combinada de catarata e glaucoma desde a década de 90 . Apresenta vantagem sobre a TREC isolada por permitir uma reabilitação visual mais rápida e um retorno as atividades habituais mais precocemente. ${ }^{(1)} \mathrm{O}$ uso adjunto da MMC reduz a queda da PIO durante a cirurgia do glaucoma, porém traz um risco maior de complicações tardias. ${ }^{(9)}$ Apesar da EPNP não ser superior a TREC no controle da PIO ela reduz as complicações tanto no intraoperatório como no pós-operatório. O uso de implantes esclerais mostra um aumento da filtração e uma redução da fibrose, porém aumenta o custo da cirurgia o que em algumas ocasiões poderia restringir a sua utilização. ${ }^{(5)} \mathrm{A}$ decisão por uma cirurgia combinada de catarata e glaucoma depende de vários fatores individuais incluindo a queda da AV, PIO alvo, estágio do glaucoma, idade e expectativa de vida. ${ }^{(4)}$ Já a FACOEPNP é considerada uma técnica eficaz de cirurgia combinada de catarata e glaucoma, pois proporciona uma redução importante da PIO e da necessidade de tratamento antiglaucomatoso, por apresentar menor hiperfiltração evitando a descompressão brusca que se produz na TREC. Por outro lado, a realização da iridectomia pode favorecer um menor risco da hifema e uveíte serofibrinosa. ${ }^{(1)}$

O presente estudo mostra que a FACOEPNP obteve uma importante redução da PIO durante o seguimento pós-operatório, onde a PIO inicial média pré-operatória foi de $20,1 \mathrm{mmHg}(\mathrm{dp} 4,7)$ tendo uma redução média de $-6,2$ (dp 5,1) com p<0,001. Gianoli após 12 meses de estudo obteve uma redução da PIO de 26,5 $\mathrm{mmHg}$ para $14,8 \mathrm{mmHg} .{ }^{(10)}$ Negrete em seu estudo também observou redução estatisticamente significante da 
Tabela 1

FACOEPNP X FACOTREC - Grupos

\begin{tabular}{llll}
\hline Variáveis & \multicolumn{2}{c}{ Grupo } & \\
\cline { 2 - 3 } & $\begin{array}{l}\text { FACOEPNP } \\
(\mathrm{n}=98)\end{array}$ & $\begin{array}{l}\text { FACOTREC } \\
(\mathrm{n}=50)\end{array}$ & Valor de p \\
Idade (anos) & $\mathrm{n}=83$ & $\mathrm{n}=44$ & \\
Média (dp) & $68,9(9,8)$ & $71,6(10,5)$ & \\
Mediana & 69 & 71 & 0,153 \\
Mínimo - Máximo & $39-92$ & $48-99$ & \\
Sexo - n (\%) & $\mathrm{n}=83$ & $\mathrm{n}=44$ & \\
Feminino & $49(59,0)$ & $28(63,6)$ & \\
Masculino & $34(41,0)$ & $16(36,4)$ & \\
AV LogMAR Pré & $0,80(0,60)$ & $0,64(0,50)$ & \\
Média (dp) & 0,6 & 0,4 & 0,067 \\
Mediana & $0,0-3,0$ & $0,0-2,0$ & \\
Mínimo - Máximo & & & \\
AV LogMAR Pós & $0,35(0,61)$ & $0,33(0,56)$ & \\
Média (dp) & 0,2 & 0,2 & 0,924 \\
Mediana & $0,0-3,0$ & $0,0-3,0$ & \\
Mínimo - Máximo & & & \\
AV LogMAR Variação (Pós - Pré) & $-0,45(0,51)$ & $-0,31(0,59)$ & \multirow{2}{*}{0,099} \\
Média (dp) & $-0,3$ & $-0,2$ & \\
Mediana & $-2,0-2,0$ & $-1,9-1,7$ & \\
Mínimo - Máximo & & & \\
\hline
\end{tabular}

Tabela 2

FACOEPNP X FACOTREC - Valores de PIO pré e pós-cirurgia

\begin{tabular}{|c|c|c|c|}
\hline \multirow[t]{2}{*}{ Variáveis } & \multicolumn{2}{|c|}{ Grupo } & \multirow[b]{2}{*}{ Valor de $\mathrm{p}$} \\
\hline & $\begin{array}{l}\text { FACOEPNP } \\
(\mathrm{n}=98)\end{array}$ & $\begin{array}{l}\text { FACOTREC } \\
(\mathrm{n}=50)\end{array}$ & \\
\hline \multicolumn{4}{|l|}{ PIO Pré (mmHg) } \\
\hline Média (dp) & $20,1(4,7)$ & $20,8(4,2)$ & 0,190 \\
\hline Mediana & 20 & 20,5 & \\
\hline Mínimo - Máximo & $12-48$ & $14-34$ & \\
\hline \multicolumn{4}{|l|}{ PIO Pré - n (\%) } \\
\hline$<21 \mathrm{mmHg}$ & $65(66,3)$ & $27(54,0)$ & 0,144 \\
\hline$\geq 21 \mathrm{mmHg}$ & $33(33,7)$ & $23(46,0)$ & \\
\hline \multicolumn{4}{|l|}{ PIO Pós (mmHg) } \\
\hline Média (dp) & $13,8(2,9)$ & $14,2(3,3)$ & \\
\hline Mediana & 14 & 14 & 0,376 \\
\hline Mínimo - Máximo & $7-22$ & $6-22$ & \\
\hline \multicolumn{4}{|l|}{ PIO Pós - n (\%) } \\
\hline$<21 \mathrm{mmHg}$ & $97(99,0)$ & $48(96,0)$ & 0,264 \\
\hline$\geq 21 \mathrm{mmHg}$ & $1(1,0)$ & $2(4,0)$ & \\
\hline \multicolumn{4}{|c|}{ PIO Variação (Pós - Pré) (mmHg) } \\
\hline Média (dp) & $-6,2(5,1)$ & $-6,6(5,3)$ & \\
\hline Mediana & -5 & -5 & 0,856 \\
\hline Mínimo - Máximo & $-31-4$ & $-22-3$ & \\
\hline \multicolumn{4}{|l|}{ Méd Pós - n (\%) } \\
\hline 0 & $76(77,6)$ & $42(84,0)$ & \\
\hline 1 & $14(14,3)$ & $4(8,0)$ & 0,398 \\
\hline 2 & $8(8,2)$ & $4(8,0)$ & \\
\hline \multicolumn{4}{|l|}{ Sucesso - n (\%) } \\
\hline Sucesso absoluto & $75(76,5)$ & $41(82,0)$ & \\
\hline Sucesso relativo & $22(22,4)$ & $7(14,0)$ & 0,253 \\
\hline Não & $1(1,0)$ & $2(4,0)$ & \\
\hline
\end{tabular}


PIO em todas as visitas de pós-operatório; a redução média da PIO foi de $8,63 \mathrm{mmHg}(\mathrm{dp} 8,55)$ com 12 meses de seguimento correspondendo a uma redução de $32,78 \%$. $^{(1)} \mathrm{O}$ grupo da FACOEPNP apresentou AV LogMar pré-média de $0,80(\mathrm{dp} 0,60)$ evoluindo para $\mathrm{AV}$ LogMar pós-média de 0,35 (dp 0,61 ) com variação média da AV LogMar de $-0,45$ (dp 0,51) com $p<0,001$. Segundo Luke seu estudo mostrou uma melhora na AV LogMar em 1,6 linhas (dp 2,4).(11)

Em relação ao grupo submetido à FACOTREC a PIO pré-média era de $20,8 \mathrm{mmHg}(\mathrm{dp} \mathrm{4,2)}$ e após 12 meses de seguimento a PIO pós-média foi de $14,2 \mathrm{mmHg}$ (dp 3,3) com $\mathrm{p}<0,001$. Gianoli mostrou PIO pré-média de $25,9 \mathrm{mmHg}$, caindo para $14,8 \mathrm{mmHg}$ no pós-operatório com o mesmo procedimento cirúrgico. ${ }^{(10)}$ Jurowski em 6 meses após FACOTREC obteve uma queda de pressão de 62,9\%.(12) Em estudo realizado por Luke com PIO inicial média de $26,5 \mathrm{mmHg}(\mathrm{dp} 7,8)$ obteve PIO média no pós-operatório de $14,4 \mathrm{mmHg}(\mathrm{dp} 4,0)$ com $\mathrm{p}<0,001$.(13) A média de variação da AV LogMar no grupo FACOTREC foi de -0,31 (dp 0,59), apresentando AVLogMar média pré de 0,64 (dp 0,50) com AV Logmar média pós de 0,33 ( dp 0,56) com $\mathrm{p}<0,001$.

Em nosso estudo 76 pacientes ( $77,6 \%$ ) não necessitaram de medicação antiglaucomatosa no pós-operatório, 14 $(14,3 \%)$ necessitaram de 1 uma droga antiglaucomatosa e 8 $(8,1 \%)$ necessitaram de 2 medicações no grupo submetido à FACOEPNP. No grupo da FACOTREC, 42 pacientes (84,0\%) não necessitaram de medicação antiglaucomatosa, $4(8,0 \%)$ necessitaram de 1 medicação e 4 (8,0\%) necessitaram de 2 drogas no pós-operatório. Segundo Yuen, o número de medicações antiglaucomatosas para o controle da PIO diminuiu de 3,28 para 0 após 6 meses de seguimento. ${ }^{(14)}$ Em outro trabalho, Yeun obteve significante redução no número de medicações antiglaucomatosas oscilando de 2,9 (dp 0,8) para $1,0(\mathrm{dp} 1,2){ }^{(15)}$

Na FACOEPNP a taxa de sucesso absoluto foi de $76,5 \%$ ( 75 pacientes) e $22,4 \%$ ( 22 pacientes) obtiveram sucesso relativo. Já na FACOTREC, 82,0\% (41 pacientes) obtiveram sucesso absoluto e $14,0 \%$ (7 pacientes) sucesso relativo após 12 meses de seguimento. De acordo com os mesmos critérios de sucesso absoluto e relativo adotados pelo trabalho, Gianoli obteve sucesso absoluto em $59 \%$ de seus pacientes e sucesso relativo em $80 \%$ após 12 meses de cirurgia.

Não foi encontrada diferença estatisticamente significante entre os grupos quanto à distribuição dos resultados de eficácia dos procedimentos $(\mathrm{p}=0,253)$, assim como demonstrado em estudos realizados por Gianoli. ${ }^{(10,16)}$

\section{ConClusão}

Podemos concluir que ambas as cirurgias resultam em uma queda da PIO similar e mesmo resultado em relação a melhor AV.Cabe ao cirurgião optar pela técnica a ser utilizada, comparando as possíveis complicações e a curva de aprendizado.

\section{Abstract}

Purpose: the aim of the present study is to compare the intraocular pressure (IOP) and visual acuity (VA) after combined surgery: phacoemulsification with non penetrating deep sclerectomy and phacoemulsification with trabeculectomy. Methods: this is an observacional and retrospective study by chart review. We selected 148 eyes from 127 patients from Tadeu Cvintal Ophthalmolgy Institute during January 1998 thru January 2007. All patients had primary angle glaucoma and cataract. Seventy seven patients were female, 49 (59\%) had phacoemulsification with non penetrating deep sclerectomy and 28 (63,6\%) had phacoemulsification with trabeculectomy. Fifty patients were male, 34 (41\%) had phacoemulsification with non penetrating deep sclerectomy and $16(36,4 \%)$ phacoemulsification with trabeculectomy. The patients had IOP (Goldmann) and VA (LogMar) measured before surgery and one year after surgery, also the number of glaucoma medications that patients had to drop after surgery. Results: the groups were equals comparing VA before $(p=0,067)$, and after VA $(p=0,924)$ with variation $V A(p=0,099)$. The mean VA in the group that had phacoemulsification with trabeculectomy was $0,31(d p=0,59)(p<0,0001)$, and the mean variation in the group that had phacoemulsification with non penetrating deep sclerectomy was $-0,45(d p 0,51)$ with $p, 0,001$. The $I O P$ mean variation in the patients that had phacoemulsification with non penetrating deep sclerectomy $-6,2(d p=5,1)$ had stastistically difference $(p<0,001)$ and the mean variation of IOP in the patients that underwent phacoemulsification with trabeculectomy was $-6,6(d p=$ $5,3)(p<0,001)$. There was no statistically difference between the groups evaluating the glaucoma medications. Conclusion: non penetrating deep sclerectomy combined with phacoemulsification causes IOP reduction similar phacoemulsification with trabeculectomy with the same result evaluating $V A$.

Keywords: Open-angle glaucoma/surgery; Trabeculectomy; Sclera/surgery; Phacoemulsification; Intraocular pressure; Visual acuity; Combined modality therapy 


\section{ReFERÊNCIAS}

1. Muñoz Negrete FJ, Rebolleda G, Noval S. [Non-penetrating deep sclerectomy combined with phacoemulsification. Results and complications]. Arch Soc Esp Oftalmol. 2003;78(9):499-506. Spanish.

2. Lyle WA, Jin JC. Comparision of a 3- and 6-mm incision in combined phacoemulsification and trabeculectomy. Am J Ophthalmol 1991; 111(2):189-96.

3. Lachkar Y, Hamard P. Nonpenetrating filtering surgery. Curr Opin Ophtalmol. 2002; 13(2):110-5.

4. Funnell CL, Clowes M, Anand N. Combined cataract and glaucoma surgery with mimomycin C: phacoemulsificationtrabeculectomy compared to phacoemulsification-deep sclerectomy. Br J Ophthalmol 2005; 89: 694-698.

5. Moreno-López M, Pérez-Alvarez MJ. [Short- and mediumterm intraocular pressure lowering effects of combined phacoemulsification and non-penetrating deep sclerectomy without scleral implant or antifibrotics]. Arch Soc Esp Oftalmol. 2006;81(2):93-100. Spanish.

6. Mermoud A, Karlen ME, Schnyder CC, Sickenberg M, Chiou AG, Hédiguer SE, et al. Nd: Yag goniopuncture after deep sclerectomy with collagen implant. Ophthalmic Surg Lasers. 1999; 30(2):120-5.

7. Lama PJ, Fechtner RD. Antifibrotics and wound healing in glaucoma surgery. Surv Ophthalmol. 2003; 48(3):314-46.

8. Mandia Junior C, Kasahara N, Seixas FS, Paolera MD, Almeida GV, Cohen R. Comparacão a longo prazo entre a facectomia extracapsular combinada à trabeculectomia e à facotrabeculectomia. Arq Bras Oftalmol. 2002; 65(6):641-4.

9. Anand S, Anand N. Combined phacoemulsification and deep sclerectomy (PDS) with intraoperative mitomycin C (MMC) augmentation. Eye (Lond). 2008; 22(8):1040-9.
10. Gianoli F, Mermoud A. [Cataract-glaucoma combined surgery: comparison between phacoemulsification combined with deep sclerectomy, or trabeculectomy]. Klin Monbl Augenheilkd. 1997;210(5):256-60. French.

11. Lüke C, Dietlein TS, Lüke M, Konen W, Krieglstein GK. Phacotrabeculotomy combined with deep sclerectomy, a new technique in combined cataract and glaucoma surgery: complication profile. Acta Ophthalmol Scand. 2007;85(2):143-8.

12. Jurowski P, Gos R. [Effectiveness of the combined surgical treatment for glaucoma and cataract]. Klin Oczna. 2005;107(4-6):212-6. Polish.

13. Lüke C, Dietlein TS, Lüke M, Konen W, Krieglstein GK. A prospective trial of phaco-trabeculectomy combined with deep sclerectomy versus phaco-trabeculectomy. Graefes Arch Clin Exp Ophthalmol 2008;246(8):1163-8.

14. Yuen NS. Early results of modified nonpenetrating deep sclerectomy and phacoemulsification in the treatment of open angle glaucoma and cataract. Eur J Ophthalmol. 2009;19(1):72- 9.

15. Yuen NS, Chan OC, Hui SP, Ching RH. Combined phacoemulsification and nonpenetrating deep sclerectomy in the treatment of chronic angle-closure glaucoma with cataract. Eur J Ophthalmol. 2007;17(2):208-15.

16. Gianoli F, Schnyder CC, Bovey E, Mermoud A. Combined surgery for cataract and glaucoma: phacoemulsification and deep sclerectomy compared with phacoemulsification and trabeculectomy. J Cataract Refract Surg. 1999;25(3):340-6.

\section{Endereço para correspondência: \\ Juliana Almodin \\ Rua Xavier Curado, $n^{\circ} 351$ - apto 131 - Ipiranga \\ CEP 04210-100 - São Paulo (SP), Brasil \\ E-mail: Juliana_almodin@hotmail.com}

